\title{
RF Optimization for Call Setup and Analysis of GSM Network Using Agilent Tools
}

\author{
Syed Sazad ${ }^{1}$, Banani Talukdar ${ }^{2}$, Rajat Paul ${ }^{3}$ \\ Department of Electronics and Communication, Sikkim ManipalInstitude of Technology, Majhitar, Rangpo, East Sikkim-737136, India
}

\begin{abstract}
The Call Set up Success Rate, successful handovers, and maintaining the quality of call are most important features used by all mobile operators. So, it is extremely necessary and mandatory to identify possible means to measure these parameters and eliminate the existing problems in a GSM network. Therefore the different operators can use various type of optimization process. How to optimize the GSM coverage area successfully along with better service is the real challenge. In this paper the main motive is to identify the causes of call setup failures in a GSM service test area and necessitate steps to increase the call success rate using RF optimization. RF optimization consists of drive testing, post processing, data analysis, recommendations and action steps. Optimization will be continuous and iterative process of improving network quality. By successful optimization, the Quality of Service, reliability and availability of RF Coverage area is highly improved. $R F$ optimization is used to make proposals on how operators can optimize radio resources as well as provide the required of service quality to the subscribers. Since call drop and call failure are the major problems in the GSM networks. So this RF optimization technique helps to solve this problem.
\end{abstract}

Keywords: RF optimization, GSM network, Drive test, Call drop, Call failure, Post processing, urban and rural areas, Quality of service (Qos), Coverage.

\section{Introduction}

As we have seen call rates and the call drop are the major problems in the GSM network. So this RF optimization is done in order to reduce such problems. In present wireless communication systems the customers' demands the quality of service. GSM Network is comprised of a mobile Station (MS) which is connected to the Base Transceiver Station (BTS) via air interface. The performance of GSM network is mainly based on radio network planning and optimization. Due to increasing subscribers, the changing environments, rapid network expansion exceeding initial projections, capacity limitations due to lack of frequency resources and subscribers mobility profile changing, we need a continuous radio network planning (RNP) and Optimization process that is required as the network evolves. Radio frequency network optimization (RNO) teams play a very significant and vital role in optimizing an operational network to meet the ever increasing demands from the end users. A system with good coverage will always be superior to a system with less good coverage. Planning of wireless networks is vital if operators wish to make full use of the existing investments. It deals with a practical approach of radio network planning process for efficient usage of GSM network. The key performance indicator (KPI) and drive test report of operator are used to make proposals on how operators can optimize radio resources as well as provide the required Qos to the subscribers. This study would help to plan operators to enhance coverage, improve quality and increase capacity in the days to come. The frequency bandwidth division for the various services providers and also explains about the frequency reuse for the effective usage. This gives the description about the Traffic Channel, which required for the communication between the users and the accessing the services from the network. This also explains about the interface between the GMS and internet access facility in mobile using GPRS.

\section{RF Optimization}

The mobile communication aims to offer anytime and anywhere communications between any objects. GSM network performance and QoS evaluation are the most important steps for the mobile operators as the revenue and customer satisfaction is directly related to network performance and quality. Radio frequency network optimization (RNO) teams play a very significant and vital role in optimizing an operational network to meet the ever increasing demands from the end users.RF Optimization is a very important process in any service providers operating lifecycle. By gathering and analysing network data and revising network parameters Cellular communication achieved by using proper RF Planning and Optimization. As we move further ahead the need for better technologies and reliability of services, integration and cost effective solutions have become a necessity for service providers. If the optimization is successfully performed means you achieve the QoS, reliability, Optimization involves monitoring, verifying and improving the performance of the radio network. It starts somewhere near the last phase of radio network planning, i.e. during parameter planning. A cellular network covers a large area and provides capacity to many people, so there are lots of parameters involved that are variable and have to be continuously monitored and corrected. Apart from this, the network is always growing through increasing subscriber numbers and increases in traffic. This means that the optimization process should be on-going, to increase the efficiency of the network leading to revenue generation from the network. 
International Journal of Science and Research (IJSR)

ISSN (Online): 2319-7064

Index Copernicus Value (2013): 6.14 | Impact Factor (2014): 5.611

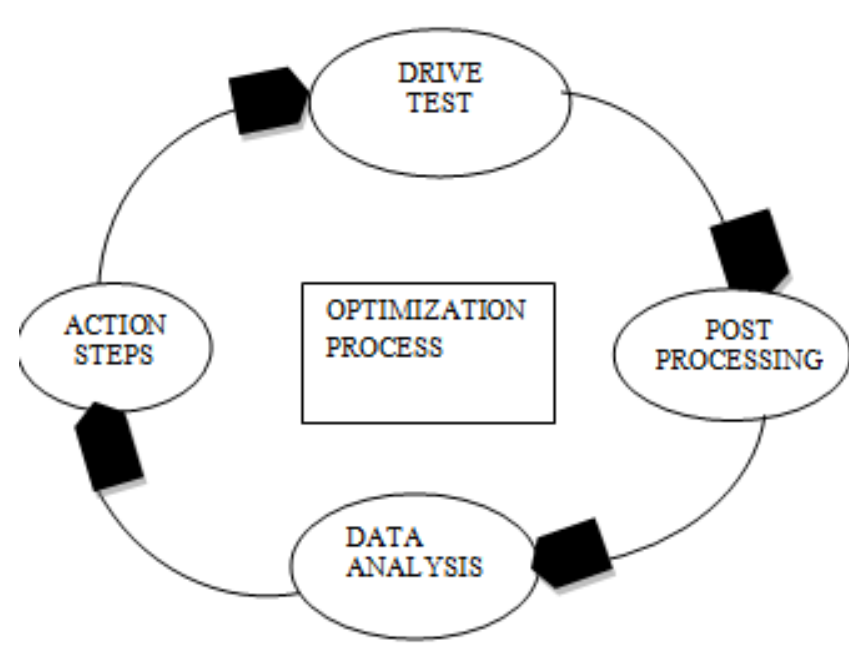

Figure 1: RF optimization process

\section{Optimization Process}

\subsection{Drive Test}

Agilent's JDSU E6474A v15.2 is the tool we have used for this work. Agilent's E6474A drive test tool has revolutionized and simplified end to end troubleshooting. The software allows users to correlate signalling procedures from the air interface and radio access network interfaces in a single view to detect and troubleshoot problems from the mobile phone to the network. The drive test helps in Automatic correlation of data collected from both the radio and network interfaces to find end-to-end performance issues more easily. The quality of the network is ultimately determined by the satisfaction of the users of the network, the subscribers. Drive tests give the 'feel' of the designed network as it is experienced in the field. The testing process starts with selection of the 'live' region of the network where the tests need to be performed, and the drive testing path.In urban areas, coverage is generally found to be less at the farthest parts of the network, in the areas behind high buildings and inside buildings. This leads to an immediate scrutiny of the antenna locations, heights and tilt. The problems are usually sorted out by moving the antenna locations and altering the tilting of the antennas. If optimization is being done after a long time, new sites can also be added. Coverage also becomes critical in rural areas, where the capacity of the cell sites is already low. Populated areas and highways usually constitute the regions that should have the desired level of coverage. For highway coverage, additions of new sites may be one of the solutions.

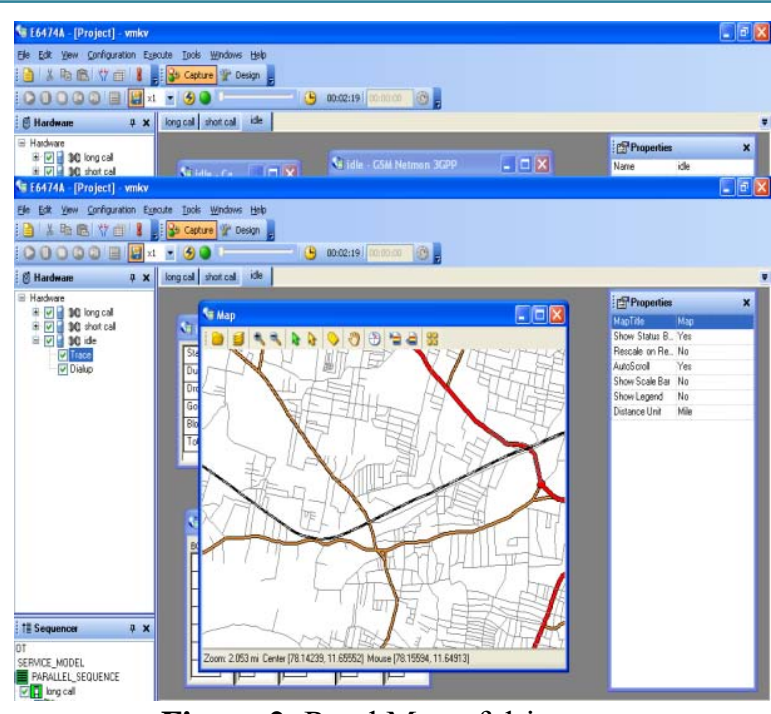

Figure 2: Road Map of drive test

The data as per the requirement are observed and recorded. The data is analysed for performance. The design is validated and based on the data analysis, the percentage of good network and bad network is concluded. For the test network, the good design and bad design percentages are calculated based on the integration of test samples. Further, the causes for the bad design are contemplated and the total outcome is broken into fragments for in-depth analysis of the root cause. These fragments are compared against the standard values and wherever possible the design is improved .The improvisation of design is based on the optimised solutions suggested. The analysis of the drive test is depicted in a graphical form, like a bar chart and conclusions are derived based on the charts.

\subsection{Post processing}

Actixanalyzer is a software application from the Agilent that is normally used for the Network performance optimization. The post processing is RF optimization is done so as to provide feature testing. The GSN network may have many problem in the cellular networks such as call drop and call fail so this process helps us to determine the problems and the find the appropriate solution for the desired problem. This process also involves the competitive analysis of the different networks under different conditions. The post processing involves the one set of calls namely short calls and long calls. As we know the signal is received from the base station to the user. The received signal is determined by the base station. In cellular communication the frequency reuse is the most important parameter to be considered When the user is communicating while moving at the high speed, then the network should be more effective to perform a handover between the each cell. If the user is at high speed, the network tower changes periodically and if handover is not performing at the threshold time then it results in call drop and call failure. The figure 2 and 3 shows the road map of GSM and the display of the proposed network. 


\section{International Journal of Science and Research (IJSR) \\ ISSN (Online): 2319-7064}

Index Copernicus Value (2013): 6.14 | Impact Factor (2014): 5.611

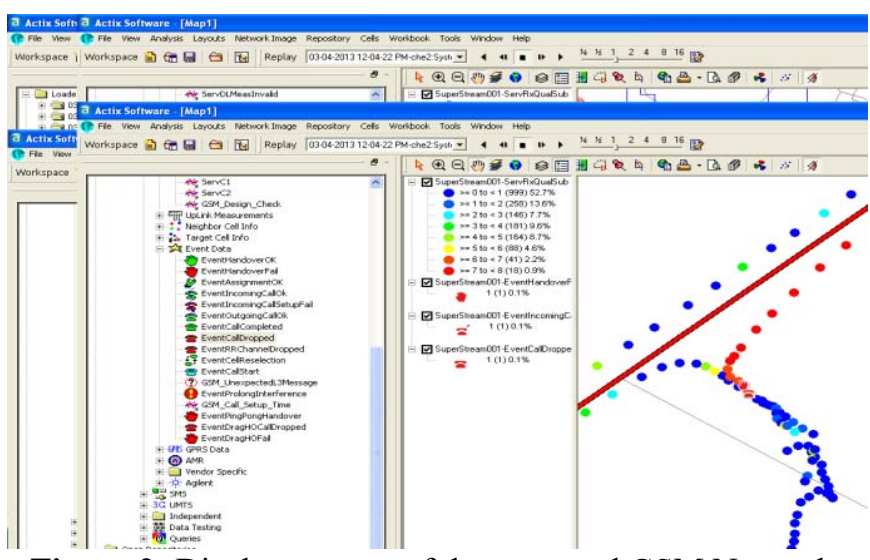

Figure 2: Display on map of the proposed GSM Network

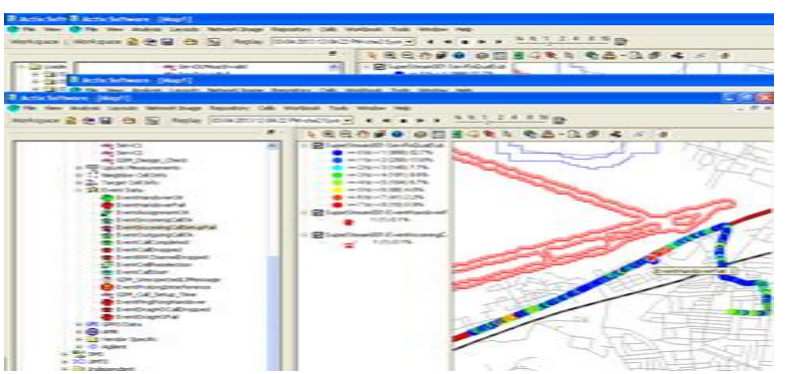

Figure 2: Plotting the event data of the proposed Network.

\subsection{Data analysis and Action steps}

From the GSM line chart window we have selected the corresponding line chart for long calls, short calls and idle calls. The line chart provide complete details of the serving cell BSIC, HSN, MAIO, Rx Qual, cell Id.Fig 3. Shows the analysis of the GSM network.

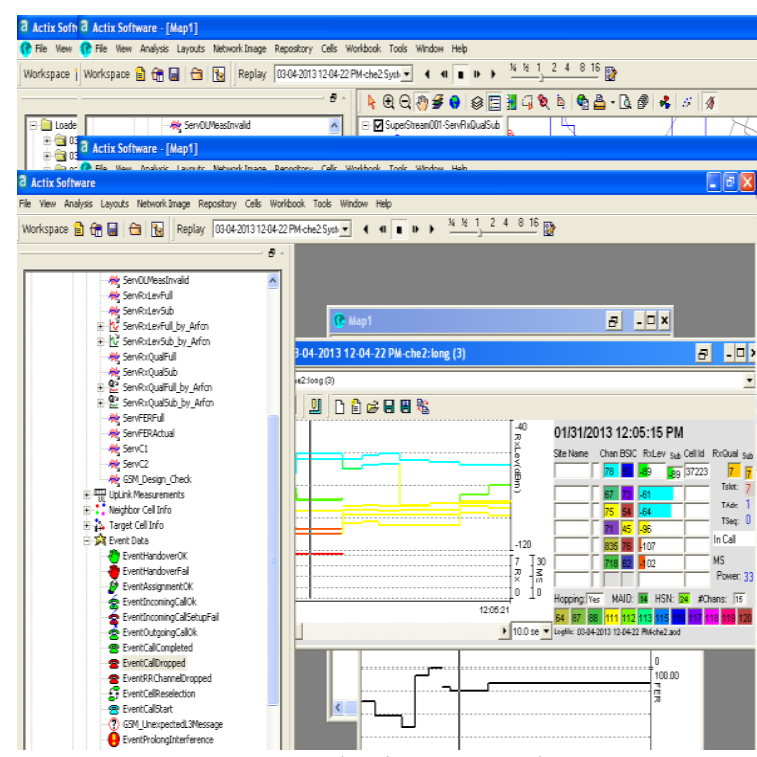

Figure 3: Plotting GSM charts

In the above figure, it is seen that the red line indicates the continuity of long call, the corresponding channel and BSIC are shown in the side column. Different channels are marked using different colours in the line graph. From the figure, the call drop is indicated by the termination of the red line. Moment the red line terminates, all the parameters such as RxQual, HSN, and MAIO are also terminated. This chart tells us about the time the call was dropped, the serving cell parameters, neighbour cells, BSIC. For short calls the calls are generated periodically for short duration of time say 10 seconds. Idle call does not perform any calls but remain idle.

\section{Call Setup in GSM Network}

The successful call set up consists of two procedures. The simplified description of these procedures is provided in the next text in such a way that the focus is only on the parts necessary to understand the philosophy of Call Set up Success Rate calculation correctly. First procedure is Immediate Assignment procedure which is used to create a signalling connection between the Mobile station (MS) and the network. It can be initiated only by the MS sending a CHANNEL REQUEST message on the Random Access channel (RACH) to the BTS that it requires a signalling channel (SDCCH). This message contains the information field establishment cause and random reference. The establishment cause gives the reason why the MS is requesting a SDCCH. Then comes the next signalization between the MS and network in order to activate the signalling channel, recognize the service being requested by the MS, etc. The successful seizure of SDCCH is acknowledged by sending the Establish Indication message from MS to BTS and then to BSC. Further coordination procedure (authentication, ciphering etc.) are now performed on the SDCCH. Second procedure is Assignment procedure which is used to occupy a radio resource (speech channel). The MSC is initiator of this procedure. The MSC sends an ASSIGNMENT REQUEST message to the BSC requesting the assignment of a radio resource (RR). Then comes next signalization between BTS and BSC in order to allocate and activate a suitable RR (Traffic channel - TCH).If the TCH is successfully seized by MS, the BSC sends the ASSIGNMENT COMPLETE message

\section{Conclusion}

Due to the mobility of subscribers and complexity of the radio wave propagation, most of the network problems are caused by increasing subscribers and the changing environment. RF Optimization is a continuous process that is required as the network evolves. RF optimization is carried out in order to improve the network performance with the existing resources. Through RF Optimization, the service quality and resources usage of the network are greatly improved and the balance among coverage, capacity and quality is achieved. At present Drive Testing in GSM $\mathrm{RF}$ Optimization is being performing manually for the improvement of performance of the network. Instead of doing drive testing manually, there may be a scope of ANMS (Automatic Network Management System) process in which system, Drive Testing equipment can be attached to moving vehicle to serve in GSM test area and it can be monitored by the server. By using the internet, all the real time drive data can be simultaneously collected.

\section{References}

[1] RF Optimization of GSM Manual from Bharat Sanchar Nigam Limited (BSNL). 


\section{International Journal of Science and Research (IJSR) \\ ISSN (Online): 2319-7064}

Index Copernicus Value (2013): 6.14 | Impact Factor (2014): 5.611

[2] Communication system, $4^{\text {th }}$ edition, Simon Haykin, Mc Master University.

[3] Wireless Communications, Principles and Practice, $2^{\text {nd }}$ edition, Theodore S. Rappaport, Pearson Publications, 2003.

[4] Halonen T., Romero J., Melero J. GSM, GPRS and EDGE performance. John wiley and son's ltd., 2003.

[5] Intelligent Optimization of GSM Network RichaChitranshi, JyotiKushwaha, Prakash Pancholy. International Journal of Engineering Science and Innovative Technology (IJESIT) Volume 1, Issue 2, November 2012.

[6] Wireless Communications, Principles and Practice, 2nd edition, Theodore S.Rappaport, Pearson publications.

[7] Radio Frequency Optimization \&QoS Evaluation in Operational GSM Network BilalHaider, M. Zafrullah and M. K. Islam. Proceedings of the World Congress on Engineering and Computer Science 2009 Vol I WCECS 2009, October 20-22, 2009, San Francisco, USA.

\section{Author Profile}

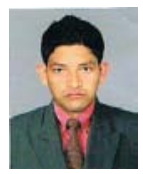

SyedSazad received the B.E degree from Vinayaka Missions University, Tamil Nadu, India. Recently he is pursuing the M.Tech degree in Digital Electronics and Advance Communication from Sikkim Manipal University. $\mathrm{He}$ has publishedseveral papers in International Journals and attended various conferences. His research area includes RF Optimization, Cognitive Radio, Wireless Communication, and Advance Digital Signal Processing, Mobile Communication, SystemVue Software and Electronics devices. Recently he is working on his thesis on Hardware Implementation of a Wi-Fi ac Transreceiver System Using Agilent Vector Signal Analyzer.

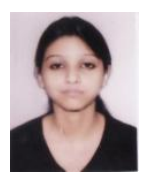

BananiTalukhdar received the B. Tech degree from Assam Don Bosco University, Assam, India. Recently she is pursuing the $M$. Tech degree in Digital Electronics and Advance Communication from Sikkim Manipal University. She has published several papers in International Journals and attended various conferences. Her research area includes RF Optimization, Nano Technology, Microwave, ADSP, Digital Electronics, Microprocessor and Mobile Communication. Recently she is working on the thesis on Simulation and Design of a Single Electron Transistor and realising the application in Digital Circuits.

Rajat Paul received the B. Tech degree from West Bengal University of Technology, West Bengal, India. Recently he is pursuing the M. Tech degree in Digital Electronics and Advance Communication from Sikkim Manipal University. His research of area includes RF Opitimization, Cognitive Radio, Wireless Communication, Analog and Digital Communication, Advance Digital Signal Processing, VLSI, Microprocessor, Satellite and Optical Communication. 